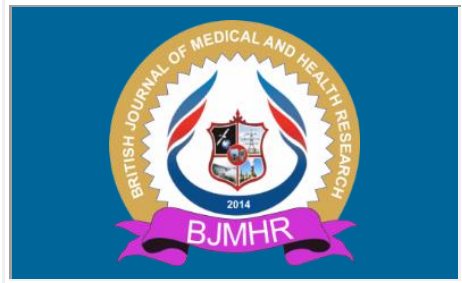

\title{
BJMHR
}

British Journal of Medical and Health Research

Journal home page: www.bjmhr.com

\section{Takayasu Arteritis: Early Diagnosis Leading to Better Outcome and Quality of Life.}

\author{
Hetal Pandya, Gill Rooppreet*, Barot Hiral \\ Department of Medicine, Smt.B.K Shah Medical Institute \& Research Centre,Sumandeep \\ Vidyapeeth,Pipariya, Vadodara-391760
}

\begin{abstract}
Takayasu arteritis is an inflammatory and stenotic disease of medium and large-sized arteries characterized by a strong predilection for the aortic arch and its branches. It has complex clinical presentation and 3 stage scheme is used for simplification with most patients presenting late delaying the diagnosis. We are reporting a case of 40 years old female patient presenting in second stage (vasculitic) of this rare disease. This report highlights the fact that diagnosing this rare type of vasculitis in early stages is critical to management and prevention of dreadful complications like large arteries aneurysm, Pulmonary Hypertension, Renal artery stenosis etc. and leading to better quality of life with less episodes of active disease.
\end{abstract}

Keywords: large vessel disease, Takayasu, adult, vasculitis, arteritis 


\section{INTRODUCTION}

Takayasu arteritis is an inflammatory and stenotic disease of medium and large-sized arteries characterized by a strong predilection for the aortic arch and its branches ${ }^{1}$.

It is an uncommon disease with an incidence rate of 1.2 -2.6 cases per million ${ }^{1}$. It is most prevalent in adolescent girls and young women and more common in Asia. With poorly understood pathogenesis, cytotoxic $\mathrm{T}$ cell mediated infiltration of the vessel walls of aorta and its branches is the most accepted postulated mechanism so far. The inflammation can be localized to portion of thoracic or abdominal aorta or can even involve the entire vessel wall. In half of the patients the abdominal aorta and pulmonary arteries are involved. ${ }^{2}$ Patients present with non-specific constitutional symptoms initially. Hence it is of utmost importance to recognize the disease in its initial stages in order to prevent irreversible organ damage and to improve the quality of life of the patient.

\section{CASE REPORT}

A 40 years old Asian female presented with pain in left hand, digits, arm and forearm and tingling sensation in left upper limb for 4 months along with fatigue, anorexia and weight loss.

Patient had history of sudden onset with gradual progression of left upper limb claudication and paraesthesia since 4 months with Raynaud's phenomenon present. She also had history of an episode of dry gangrene (reversible) of digits of 2 fingers of left hand (middle and index finger) 3 days after developing claudication which alarmed her to visit physician.

She was admitted and on her examination, she had pallor, with absent pulses in left brachial artery and left radial artery but there was signs of gangrene present. Her BP in right arm was $124 / 80 \mathrm{mmHg}$ and $102 / 70$ in left arm. She had lupus like butterfly malar rash with photosensitivity. Her systemic examination was normal.

On clinical suspicion of systemic vasculitis, work up was done which revealed severe anemia with hemoglobin of $7 \mathrm{gm} \%$, raised ESR (55) and CRP ( 7.4), ANA found negative, total leukocyte count of 18100 and platelets of 5.10 . Peripheral smear confirmed anemia of chronic disease with reticulocyte count of $2 \%$. On further investigation, her C-ANCA and PANCA were found normal. She was further investigated with left upper limb Doppler (IMAGE A) which suggested thrombosis of left ulnar artery which was later confirmed with CT angiography suggestive of mild luminal narrowing of left subclavian artery, left axillary and brachial arteries and complete occlusion of left radial artery just proximal to wrist and left ulnar artery (IMAGE B). PET Scan was done which suggested mild increased FDG uptake along upper limb vessels. (IMAGE C). Based on these finding, she was diagnosed to have Large vessel vasculitis - Takayasu arteritis. 


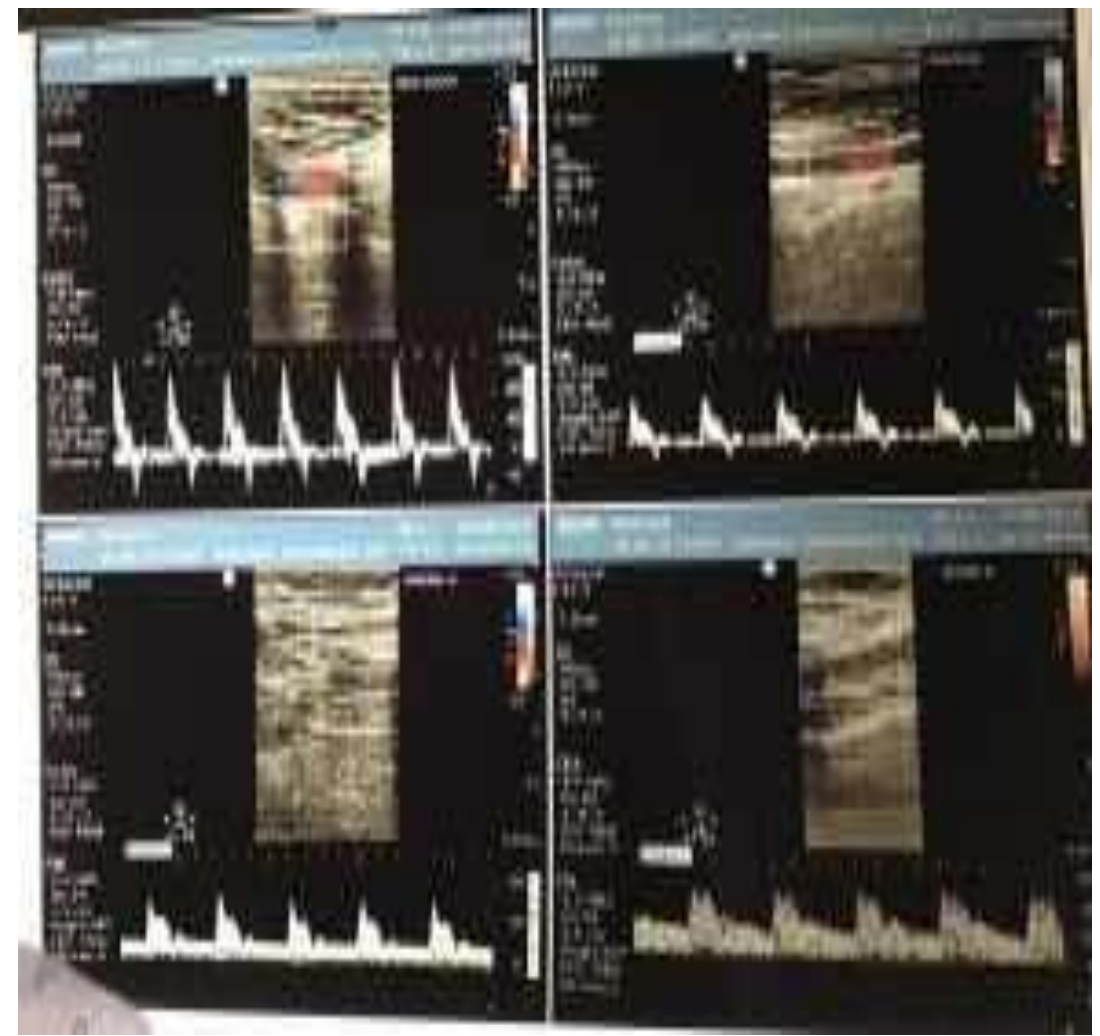

A) COLOR DOPPLER showing thrombosis of left ulnar artery

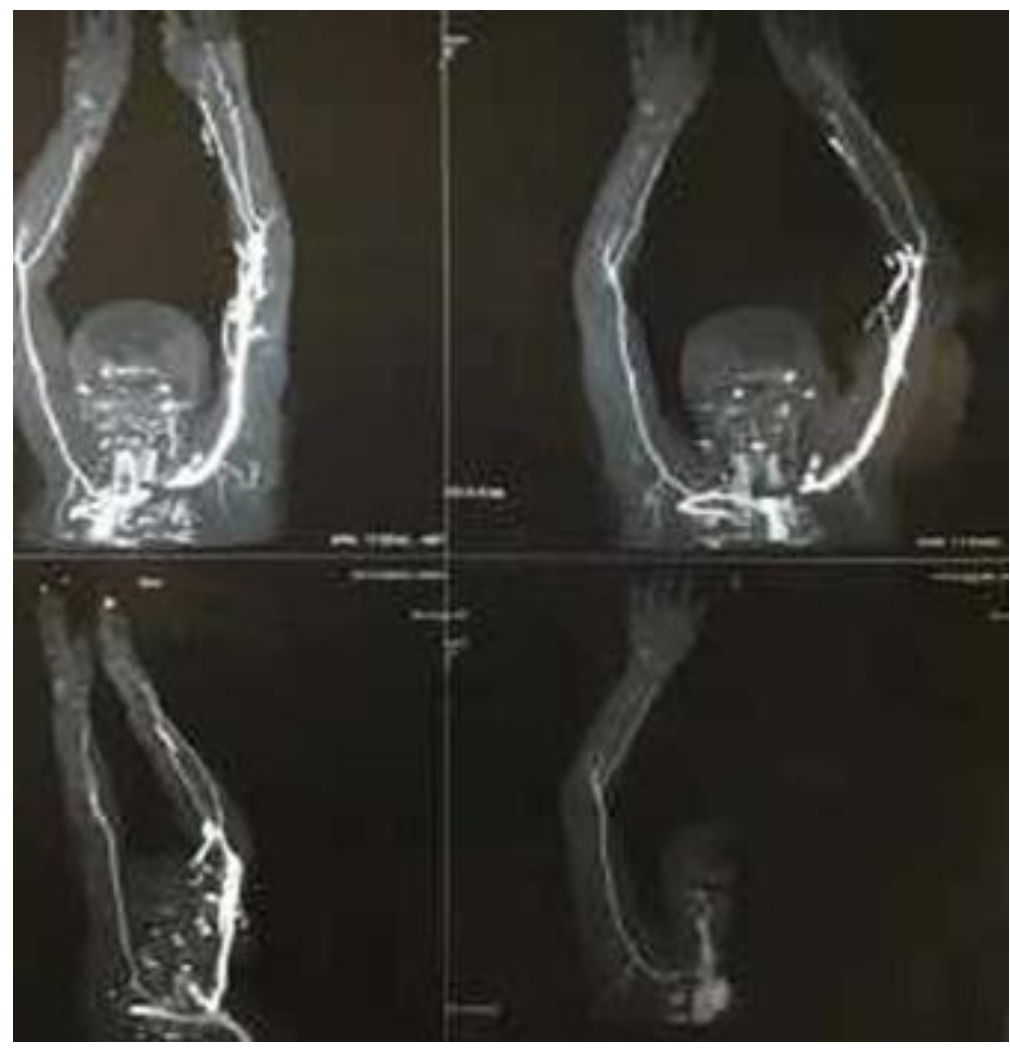

B) CTA OF UPPER LIMB VESSELS - narrowing of vessels of left side compared to right side 


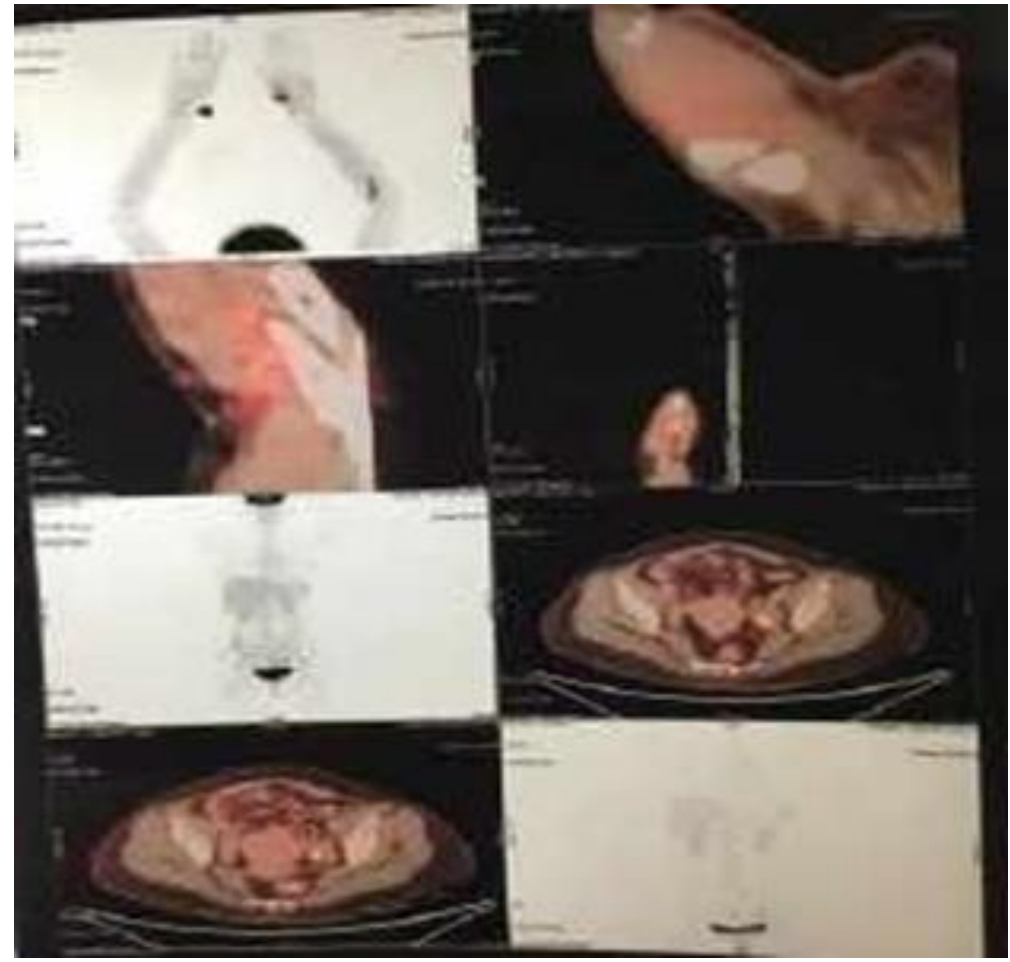

\section{C) PET SCAN - mild increased uptake of FDG in left upper limb vessels.}

She was started on corticosteroid therapy with maximum dose according to her weight. The disease activity was assessed using simplified "active disease" defining criteria's by National Institute of Health $(\mathrm{NIH})^{3}$ on follow up visit of the patient which included presence of constitutional symptoms, new-bruits, acute phase response - measured by levels of ESR and CRP. It was found that patient had no new or worsening of old symptoms, no new findings on examination and acute phase response markers were in decreasing trend, hence considering a 'stable' state of disease the corticosteroids therapy was tapered off.

\section{DISCUSSION}

Takayasu arteritis, a large vessel vasculitis most commonly seen in Asian females of age between 10-40 years ${ }^{4}$. The inflammatory process within the vessel can lead to narrowing, occlusion or dilation of involved portions of the arteries. The initial vascular lesions frequently occur in left middle or proximal subclavian artery as seen in our patient as well. With progression of the disease process, left common carotid, vertebral, brachiocephalic, right middle or proximal subclavian artery, right carotid and aorta can also be affected.

The onset of symptoms in Takayasu Arteritis is subacute leading to delay in diagnosis from months to years. Many times the first sign of disease at presentation is consequences of arterial disease when the vascular affection had already begun. Fatigue is the most common constitutional symptom along with weight loss and low - grade fever in early phase of the disease. ${ }^{5}$ As it is a rare disease, and it has progression through 3 stages, most patients present late delaying the diagnosis.

First stage 
PREVASCULITIC stage - early systemic stage with complaint of constitutional symptoms.

Second stage

Vasculitic stage -vascular inflammation occurs leading to stenosis, aneurysms and vascular pain (carotidynia).

Third stage

burned out stage - fibrosis starts and remission occurs but relapses can occur too, later on during course of disease.

As our patient presented in the second stage- the vasculitic stage with descripant blood pressure between arms. We approached this case on the basis of clinical suspicion with our findings on physical examination. As there are no specific diagnostic laboratory tests for Takayasu arteritis, but testing for acute phase reactants provide additional support for presence of systemic inflammation. The confirmatory diagnosis is by histopathological examination by biopsy which is clearly impractical. Imaging methods like MRA or CTA to evaluate arterial lumen are essential for establishing the diagnosis and determining the extent of vascular involvement. Conventional arteriography and catheter - based angiography are invasive diagnostic modalities that provides clear outlines of the lumen of involved arteries. PET scan is used to measure disease activity however its role in diagnosis is still not clear and is under study. ${ }^{6}$

American college of rheumatology classification criteria: 3 out of 6 criteria are necessary for diagnosis ${ }^{7}$ :

1. Age of 40 years or younger at disease onset

2. Claudication of extremities.

3. Decreased pulsation of one or both brachial arteries.

4. Difference of at least $10 \mathrm{mmHg}$ in systolic blood pressure between arms.

5. Bruit over one or both subclavian arteries or the abdominal aorta.

6. Arteriographic narrowing or occlusion of entire aorta, its primary branches, or large arteries in the upper or lower extremities that is not due to arteriosclerosis, fibromuscular dysplasia, or other causes.

Our patient had met 4 out of 6 criteria mentioned above, confirming the diagnosis.

The mainstay of therapy is systemic glucocorticoids. Studies have shown reduction in acute phase response markers and return of pulses after months of steroid treatment. ${ }^{8}$ The efficacy also depends on the stage of the disease when the treatment has been introduced. It has been found that $50 \%$ of patients respond to steroids as is observed in our patient too, and the rest half of the steroid unresponsive patients have been found to respond to methotrexate ${ }^{9}$. So, methotrexate is used as a steroid sparing drug, though there are other cytotoxic drugs available which can be used and with same efficacy like cyclophosphamide and azathioprine. 
As the disease process often remains active in absence of clinical symptoms, regularly scheduled investigations are highly recommended to assess the disease progression.

\section{CONCLUSION}

Takayasu arteritis, a rare type of primary systemic vasculitis, and diagnosis is done on the basis of classification criteria developed by The American college of Rheumatology and unlike in other types of vasculitis, tissue biopsy has little to no role in the diagnosis of Takayasu arteritis. This case report gives information about the approach to the early recognition of this disease, highlighting the importance of diagnosis so as to improve the quality of life of patients. As timely diagnosis not only initiates the early treatment but also allows for regular scheduled follow ups to assess disease progression and prevent irreversible damages.

\section{REFERENCES}

1. American College of Physicians (ACP). Medical Knowledge Self-Assessment Program (MKSAP-15): Rheumatology. "Systemic Vasculitis." Pg. 65-67. 2009, ACP. "American College of Physicians | Internal Medicine | ACP". Archived from the original on 2010-10-30.

2. Pulmonary artery disease in Takayasu's arteritis: angiographic findings. Yamada I, Shibuya H, Matsubara O, Umehara I, Makino T, Numano F, Suzuki S AJR Am J Roentgenol. 1992 Aug;159(2):263-9.

3. Direskeneli H, Aydin SZ, Merkel PA. Assessment of disease activity and progression in Takayasu's arteritis. Clin Exp Rheumatol. 2011 Jan-Feb. 29(1 Suppl 64):S86-91.

4. Takayasu arteritis. A study of 32 North American patients. Hall S, Barr W, Lie JT, Stanson AW, Kazmier FJ, Hunder GG Medicine (Baltimore). 1985;64(2):89.

5. Updates in Pathophysiology, Diagnosis and Management of Takayasu Arteritis.Serra R, Butrico L, Fugetto F, Chibireva MD, Malva A, De Caridi G, Massara M, Barbetta A, CannistràM, de Franciscis S Ann Vasc Surg. 2016 Aug;35:210-25. Epub 2016 May 27.

6. F-Fluorodeoxyglucose-Positron Emission Tomography As an Imaging Biomarker in a Prospective, Longitudinal Cohort of Patients With Large Vessel Vasculitis.Grayson PC, Alehashemi S, Bagheri AA, Civelek AC, Cupps TR, Kaplan MJ, Malayeri AA, Merkel PA, Novakovich E, Bluemke DA, Ahlman

7. MA Arthritis Rheumatol. 2018;70(3):439. Epub 2018 Feb 6. Arend WP; Michel BA; Bloch DA; Hunder GG; Calabrese LH; Edworthy SM; Fauci AS; Leavitt RY; Lie JT; Lightfoot RW. The American College of Rheumatology 1990 criteria for the 
classification of Takayasu arteritis .Arthritis Rheum. 1990; 33(8):1129-34 (ISSN: 0004-3591)

8. Shelhamer JH, Volkman DJ, Parrillo JE, Lawley TJ, Johnston MR, Fauci AS. Takayasu's arteritis and its therapy. Annals of internal medicine. $1985 \mathrm{Jul}$ 1;103(1):121-6.

9. Hoffman GS, Leavitt RY, Kerr GS. Treatment of Takayasu's Arteritis (TA) with methotrexate (MTX). Arthritis Rheum. 1991;34:S74.

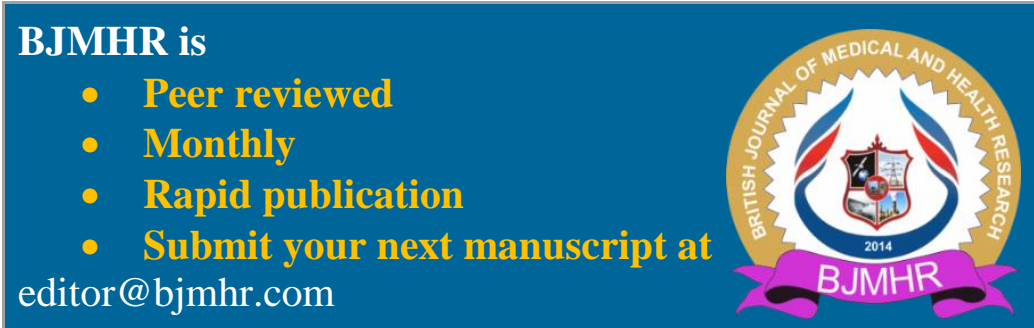

